
Coherent Sensing of Nanoscale

SUBJECT AREAS: SPECTROSCOPY

OPTICS AND PHOTONICS

QUANTUM CHEMISTRY

IMAGING TECHNIQUES

Received

5 September 2012

Accepted

25 October 2012

Published

27 November 2012

Correspondence and requests for materials should be addressed to

D.V.V. (dmitri.

voronine@gmail.com) \section{Molecular Complexes}

\author{
Dmitri V. Voronine ${ }^{1,2}$, Alexander M. Sinyukov' ${ }^{1}$ Xia Hua' ${ }^{1}$, Kai Wang' ${ }^{1}$, Pankaj K. Jha ${ }^{1,2}$, \\ Elango Munusamy' , Steven E. Wheeler', George Welch', Alexei V. Sokolov' \& Marlan O. Scully',2,3
}

${ }^{1}$ Texas A\&M University, College Station, TX 77843, ${ }^{2}$ Princeton University, Princeton, NJ 08544, ${ }^{3}$ Baylor University, Waco, TX 76798.

Nanoscale real-time molecular sensing requires large signal enhancement, small background, short detection time and high spectral resolution. We demonstrate a new vibrational spectroscopic technique which satisfies all of these conditions. This time-resolved surface-enhanced coherent anti-Stokes Raman scattering (tr-SECARS) spectroscopy is used to detect hydrogen-bonded molecular complexes of pyridine with water in the near field of gold nanoparticles with large signal enhancement and a fraction of a second collection time. Optimal spectral width and time delays of ultrashort laser pulses suppress the surface-enhanced non-resonant background. Time-resolved signals increase the spectral resolution which is limited by the width of the probe pulse and allow measuring nanoscale vibrational dephasing dynamics. This technique combined with quantum chemistry simulations may be used for the investigation of complex mixtures at the nanoscale and surface environment of artificial nanostructures and biological systems.

D etecting molecular complexes at the nanoscale presents a challenge for current state-of-the-art spectroscopy due to weak signals from small amounts of material, spectral congestion from complex mixtures and background from solvents, substrates or the molecules themselves. Two of the most commonly used techniques that provide species-specific spectroscopic signals in the form of vibrational fingerprints are surface-enhanced Raman scattering (SERS) ${ }^{1-4}$ and coherent anti-Stokes Raman scattering $(\mathrm{CARS})^{5}$ spectroscopies. In order to enhance the signal by several orders of magnitude, SERS takes advantage of the electromagnetic near-field enhancement while CARS employs molecular coherence. Several attempts have been previously made to combine these two techniques to achieve best-of-both-worlds maximum signal enhancement $t^{6-13}$. However, small enhancement factors, low spectral resolution, large background and poor reproducibility have limited application of these implementations. Overcoming these difficulties may lead to significant improvements in nanoscale sensing and new breakthrough applications in real-time label-free nano-imaging of biomolecules, detection of toxic chemicals and hazardous materials, genome sequencing, nano-catalysis and many others.

Here we have mitigated these drawbacks and demonstrated a new combination of surface and coherence enhancements by using laser pulse shaping and time-resolved detection. We applied this new time-resolved surface-enhanced coherent anti-Stokes Raman scattering (tr-SECARS) technique to investigate molecular complexes of pyridine in the vicinity of gold nanoparticles. Various molecular complexes can be formed by interaction with a nitrogen atom of pyridine (Fig. 1a). Hydrogen-bonded complexes with water cannot be excluded as water may be present in small amounts on the surface of gold. Pyridine-water complexes have been previously studied using Raman spectroscopy ${ }^{14-16}$, and pyridine was the first molecule for which SERS was demonstrated ${ }^{1}$. Single molecules and monolayers are usually the targets of research and proof-of-principle demonstrations. However, the ultimate goal is often to detect multilayer nanomolar amounts of chemicals. The near-field of plasmonic nanoparticles decays exponentially extending into a few layers of covering molecules (Fig. 1b). If these molecules are excited coherently, they may provide strong signal enhancement even from a small volume. The signal then originates both from molecular surface species and environment (including the native resonant bands of the bulk pyridine) in the vicinity of nanoparticles. 

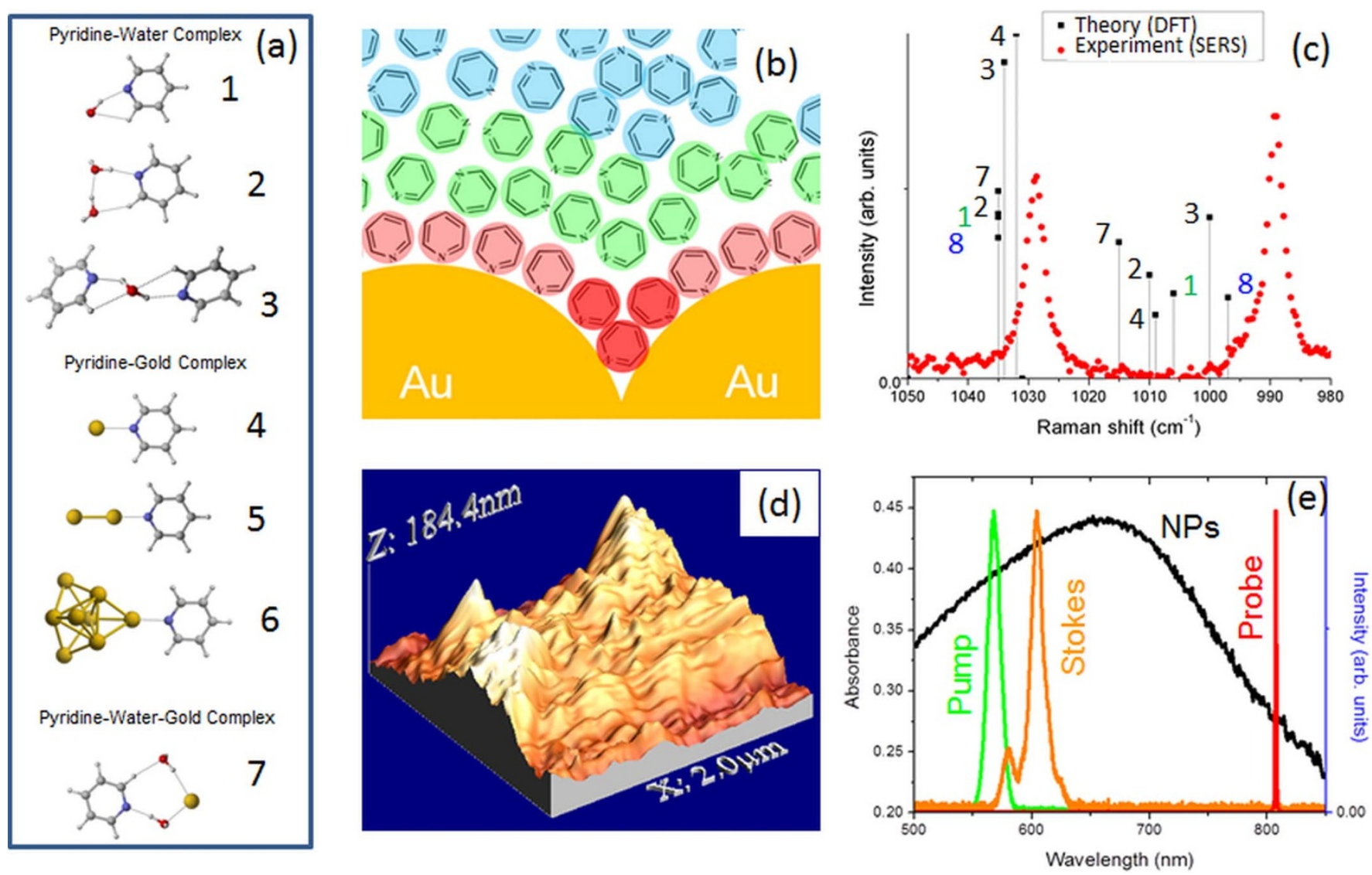

Figure $1 \mid$ (a) Equilibrium structures of various pyridine, water and gold complexes obtained from quantum chemical DFT calculations (see Supporting Material for computational details). Time-resolved SECARS identified structure 1 as the dominant species. (b) Model of pyridine molecules directly attached to (red) and in the vicinity of (green) gold nanoparticles. Bulk pyridine molecules far from the surface show no signal enhancement (blue). (c) Experimental SERS spectrum of pyridine on the surface of aggregated gold nanoparticles (red circles) and calculated Raman activities of various complexes (thin black lines) corresponding to equilibrium structures shown in (a). Lines number 8 correspond to simulated signals from pure pyridine. (d) AFM image of a surface of randomly aggregated gold nanoparticles. (e) Absorbance spectrum of the gold surface (black, NPs) overlapping with the laser pulse spectra (pump, Stokes and probe) used in the tr-SECARS experiments.

\section{Results}

A typical SERS spectrum of pyridine on aggregated gold nanoparticles is shown in Fig. 1c (red circles). The observed two strong peaks at 990 and $1030 \mathrm{~cm}^{-1}$ correspond to the ring breathing $\left(v_{1}\right)$ and triangle $\left(v_{12}\right)$ modes, respectively. These modes have been previously observed in SERS spectra on silver and gold colloid nanoparticles ${ }^{17,18}$, aggregates $^{19}$ and rough surfaces ${ }^{1}$. We used a $0.2 \mathrm{~W}$ Nd:YAG laser beam at $532 \mathrm{~nm}$ with $80 \mathrm{sec}$ signal collection time. A similar spectrum was obtained from bulk pyridine without gold NPs with resolution $\sim 3 \mathrm{~cm}^{-1}$. Our results agree with the previous reports. We used a modified sample preparation procedure (described in the Supporting Material). Briefly, a solution of colloid gold nanoparticles (NPs) with an average diameter $\sim 10 \mathrm{~nm}$ (Fig. S1A in the Supporting Material) was prepared and then deposited onto a clean glass substrate to form a multilayer aggregate of gold NPs. The resulting surface had a roughness $\sim 100 \mathrm{~nm}$ as shown in Fig. 1d (see Supporting Material). The extinction spectrum revealed a red shift and broadening of the plasmon resonance due to surface aggregation (Fig. 1e, black line). Similar randomly aggregated nanoparticles have been previously used as SERS substrates ${ }^{4}$. A drop of neat pyridine was deposited onto a gold surface and covered by a microscope glass slide to suppress evaporation. A $12 \mu \mathrm{m}$ thickness of the pyridine layer between the glass slide and the substrate was measured using an optical microscope. All experiments were performed at room temperature under ambient conditions.

To increase the signal enhancement and to decrease the collection time we implemented a time-resolved surface-enhanced CARS
(tr-SECARS) spectroscopy. In contrast to previous femtosecond SECARS techniques, our approach employs femtosecond broadband pump and Stokes pulses to excite molecular vibrational coherence and a picosecond narrowband probe pulse to generate the signal. It is based on our previously developed FAST-CARS approach to suppress the nonresonant background ${ }^{20-22}$. Here we combine the coherence enhancement of FAST-CARS with the surface enhancement of SERS. This background suppression is essential because plasmonic nanoparticles also enhance the unwanted nonresonant four-wave mixing. This has been a challenge of several recent experiments on surface-enhanced CARS spectroscopy ${ }^{7,12,13}$. The combination of these advantages allows the use of tr-SECARS to detect and investigate molecular species adsorbed onto the surface of nanoparticles in the presence of the background bulk molecules not interacting with the surface.

We performed tr-SECARS spectroscopy of the $12 \mu \mathrm{m}$ thick pyridine samples described above. The experimental setup is based on the previously described configuration ${ }^{21,22}$. A simplified scheme in Fig. 2a shows three collinear laser pulses incident on a sample of gold nanoparticles coupled to pyridine molecules. The full setup is described in the Supporting Material. Briefly, the center frequencies of the three laser beams incident on a sample were chosen to match the plasmon resonance peak of the aggregated gold NPs (Fig. 1e). The pump and Stokes are femtosecond broadband pulses at 560 and $600 \mathrm{~nm}$, respectively (FWHM $\sim 60 \mathrm{fs}$ ). The probe pulse is shaped by a variable slit of a home-built pulse shaper to have a top-hat-like spectrum at $805 \mathrm{~nm}$ (Fig. 2a). The probe pulse is thus stretched to a 

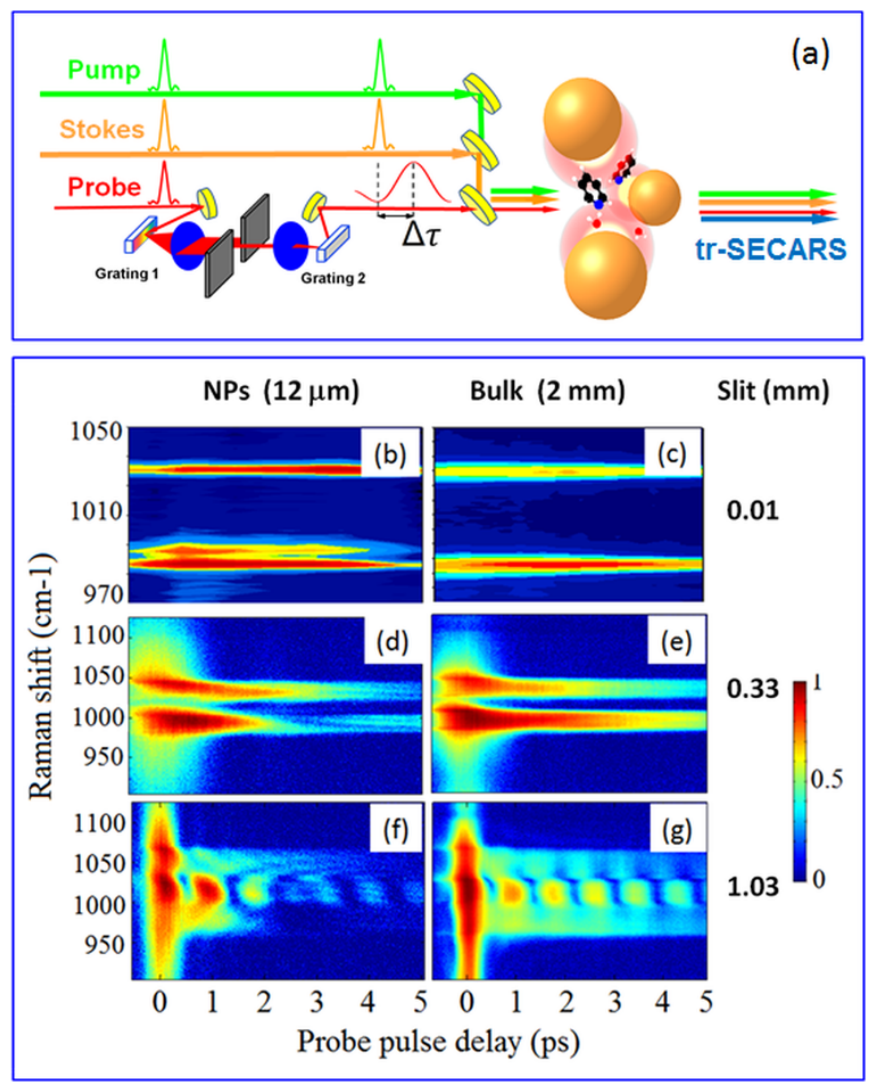

Figure $2 \mid$ (a) Simplified experimental setup of tr-SECARS spectroscopy. Three laser beams collinearly excite nanomolar amounts of pyridine in the near-field of gold nanoparticles. The pump (green) and Stokes (orange) broadband femtosecond laser pulses excite a molecular vibrational coherence which is probed by a time-delayed shaped narrowband picosecond probe pulse (red). The probe pulse is Sinc-shaped by a pulse shaper slit. The tr-SECARS signal is generated in the forward direction (blue) and collected by a spectrometer with a $0.1 \mathrm{~s}$ integration time. (b) (g) 2D spectrograms obtained by scanning the probe pulse delay. The slit of the pulse shaper determines the spectral resolution and allows resolving three and two pyridine Raman lines with (b, d, f) and without (c, e, g) nanoparticles, respectively. Three slit sizes are used $(0.01,0.33$ and $1.03 \mathrm{~mm}$ ) to demonstrate three cases on no, partial or complete spectral overlap. Spectra with (left column, NPs) and without (right column, Bulk) nanoparticles are obtained from $12 \mu \mathrm{m}$ and $2 \mathrm{~mm}$ thick layers of pyridine, respectively.

picosecond duration and acquires a temporal Sinc shape. The variable time delay $\Delta \tau$ scans the probe pulse to suppress the nonresonant background by placing the pump and Stokes pulses at the node of the Sinc shape. This optimized tr-SECARS technique combines the advantages of a broadband coherent vibrational excitation with high resolution narrowband probing and is well suited for microscopic imaging applications.

Typical two-dimensional tr-SECARS spectrograms are shown in Figs. $2 b-2 g$ with and without gold nanoparticles for different slit sizes. The signal intensity $(\log )$ is plotted as a function of the probe pulse delay $\Delta \tau$ (x-axis) and as a function of the Raman shift (y-axis). The spectrograms were normalized and leveled by subtracting a constant offset. We used low excitation energy (50, 50 and $100 \mathrm{~nJ}$ per pulse for the pump, Stokes and probe beams, respectively) to prevent optical damage of gold NPs. Samples of the $12 \mu \mathrm{m}$ pyridine layer with gold NPs show strong well-resolved signals (Figs. 2b, 2d and $2 \mathrm{f}$ ). However, no signals were obtained in the absence of NPs under similar conditions (see Fig. $3 e$ (green) and discussion below). To compare the signals with and without NPs we increased the thickness of the pyridine layer to $2 \mathrm{~mm}$ and obtained signals of similar strength as for the $12 \mu \mathrm{m}$ layer samples with NPs (Figs. 2c, $2 \mathrm{e}$ and $2 \mathrm{~g}$ ). Strong nonresonant background is observed around the zero probe pulse delay time when the pump, Stokes and probe pulses overlap. However, the molecular coherence created by the pump and Stokes pulses decays on a longer time scale than the duration of these
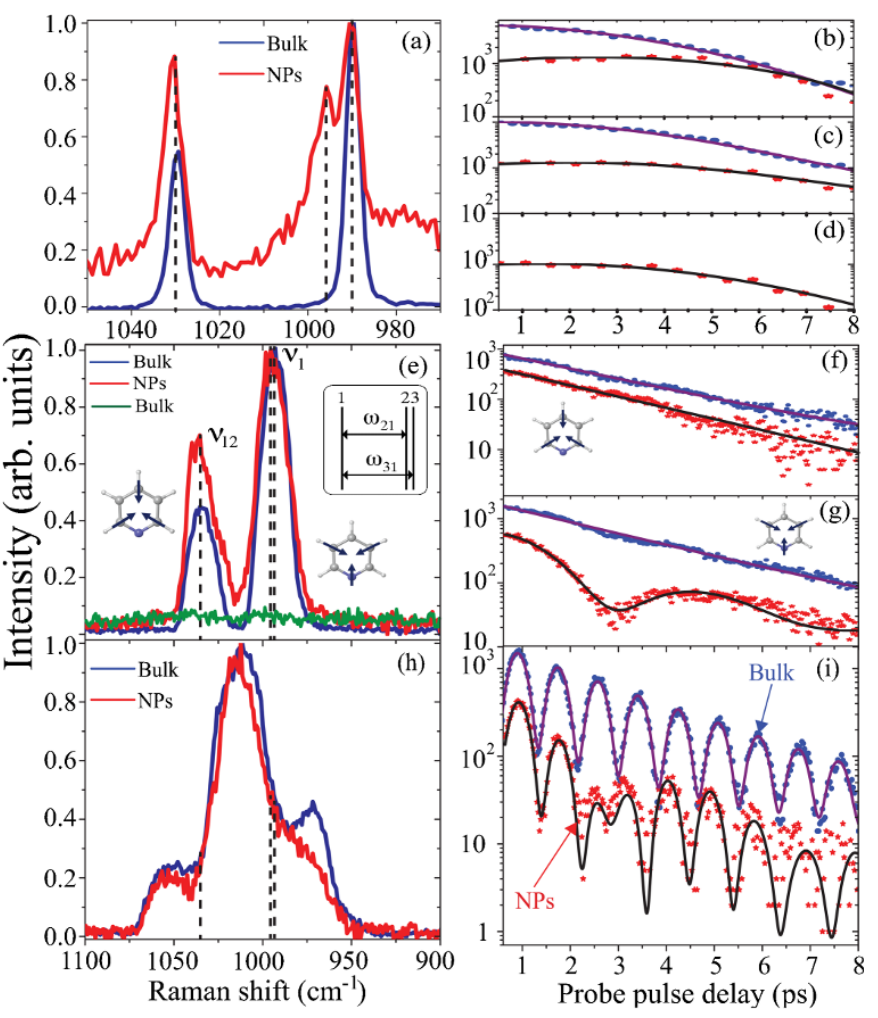

Figure 3 Normalized spectra for 1 ps delay and temporal traces of pyridine with (red) and without (blue, green) gold nanoparticles (NPs) obtained from the 2D spectrograms of Fig. 2. Signals with NPs were obtained from $12 \mu \mathrm{m}$ thick layers of pyridine on the surface of NPs. Bulk signals without NPs showed no detectable spectral features for $12 \mu \mathrm{m}$ thick samples (green, (e)). Therefore bulk signals without NPs were obtained under similar excitation conditions from $2 \mathrm{~mm}$ thick layers (blue). (a) (d) correspond to the $0.01 \mathrm{~mm}$ slit and the case of a narrow line width with little spectral overlap. Three modes (dashed) are resolved in (a) with NPs (red): at $990 \mathrm{~cm}^{-1}$ and $997 \mathrm{~cm}^{-1}$ due to the ring breathing mode of free pyridine and hydrogen-bonded pyridine-water complexes, respectively; and at $1030 \mathrm{~cm}^{-1}$ due to the triangle mode of a mixture of both species. However, only two modes are observed without NPs (blue): at 990 and $1030 \mathrm{~cm}^{-1}$ indicating no presence of water in the bulk pyridine. (b) - (d) are convolutions of the vibrational dephasing (1030, 990 and $997 \mathrm{~cm}^{-1}$ modes, respectively) with shaped probe pulse temporal profiles. (e) - (g) correspond to the $0.33 \mathrm{~mm}$ slit and intermediate case of partial spectral congestion: two spectral lines are resolved in (e) both with (red) and without (blue) NPs. The ring breathing mode of the pyridine-water complex is not resolved in the spectra (e), but can be extracted from the temporal trace $(\mathrm{g})$ where the vibrational dephasing is deconvoluted from the probe pulse shape. No detectable signals were observed without NPs under identical conditions (green) and, therefore, the amount of bulk pyridine was increased (blue). (h) and (i) correspond to the $1.03 \mathrm{~mm}$ slit where all spectral lines are congested and no modes are spectrally resolved. This latter case represents an example of femtosecond SECARS spectroscopy. Both dynamical and structural information can be obtained from the time-resolved traces (i). However all information is convoluted, and complicated fitting analysis is required. Tr-SECARS provides the advantage of using both spectral and temporal dimensions simultaneously to extract the relevant structural and dynamical information. 
pulses. Therefore the pyridine Raman signals are identified even up to 10 ps delay.

The spectral resolution is determined by the spectral width of the probe pulse which, in turn, depends on the slit size. Fig. 2 shows trSECARS spectrograms for the slit sizes of $0.01,0.33$ and $1.03 \mathrm{~mm}$ with qualitatively different Raman spectra and decay dynamics. These slits correspond to the probe pulse width of 5,22 and $65 \mathrm{~cm}^{-1}$, respectively. The spectrograms for a small slit $(0.01 \mathrm{~mm})$ revealed an additional blue-shifted line at $\sim 997 \mathrm{~cm}^{-1}$ (Fig. 2b) which was absent in bulk pyridine (Fig. 2c). The spectra with NPs were sensitive to the position of the laser focal spot on the sample and showed spatial intensity variations of this signal due to the presence of randomly distributed hot spots. For larger slits, the decreased resolution leads to quantum beating patterns due to spectral overlaps of several resonances. The samples with nanoparticles show more complicated beat patterns due to formation of pyridine complexes (Figs. $2 \mathrm{~d}$ and 2f). We carried out a detailed analysis by taking 1D traces along the $x$-and $y$-axes of these 2D spectrograms at the maxima of the Raman signals and for 1 ps probe pulse delay, respectively (Fig. 3).

Fig. 3a shows the normalized spectra of pyridine for the $0.01 \mathrm{~mm}$ slit with (red) and without (blue) gold NPs. The spectra are shown for the optimal probe delay of 1 ps which corresponds to the largest signal and smallest nonresonant background. A new blue-shifted vibrational mode of pyridine with NPs at $\sim 997 \mathrm{~cm}^{-1}$ is clearly resolved. However, the nonresonant background is not completely suppressed for this slit size due to the long duration of the probe pulse (several ps). The corresponding temporal traces for each mode are shown in Figs. $3 b-3 d$. These traces are convolutions of the probe pulse shape and exponential decays of the vibrational coherences. To extract the decay dynamics it is necessary to perform deconvolution analysis and to characterize the probe pulse shape. This can be avoided by decreasing the probe pulse duration using a larger slit width.

We consider two cases with a larger slit width of 0.33 (Figs. $3 e-3 g$ ) and $1.03 \mathrm{~mm}$ (Figs. $3 \mathrm{~h}$ and $3 \mathrm{i}$ ). The spectra in Fig. $3 \mathrm{e}$ for 1 ps probe delay show two lines both with (red) and without (blue) NPs which are different only by the amplitude ratios. Therefore, from these spectra alone it is not possible to deduce the existence of the third, blue-shifted transition due to the presence of nanoparticles. However, the temporal traces provide this information via quantum beats (Figs. $3 \mathrm{f}$ and $3 \mathrm{~g}$ ). In Fig. $3 \mathrm{f}$ both traces for the $1030 \mathrm{~cm}^{-1}$ triangle mode (with (red) and without (blue) NPs) decay monotonously. However, the $900 \mathrm{~cm}^{-1}$ ring breathing mode with NPs (red) in Fig. $3 \mathrm{~g}$ exhibits a pronounced modulation caused by a quantum beating between two resonances. In this case, the probe pulse duration is short enough for the temporal traces to directly reveal vibrational dephasing dynamics which can be easily analyzed.

Among many approaches to estimate the enhancement factor $(\mathrm{EF})^{2,3,19}$, we used direct scaling of spectral amplitudes. As described above the signals with (red) and without (blue) NPs are obtained for the $12 \mu \mathrm{m}$ and $2 \mathrm{~mm}$ layers of pyridine, respectively. Spectra of the 12 $\mu \mathrm{m}$ layers without NPs (Bulk, green) show no detectable signals (Fig. 3e). Therefore, in order to estimate the surface enhancement factor we compare signals for the $12 \mu \mathrm{m}$ (NPs) and $2 \mathrm{~mm}$ (bulk) layers. We assume that the signals with gold NPs are generated from $\sim 50 \mathrm{~nm}$ layers of pyridine which corresponds to an upper bound extension of NP near fields (Fig. 1b). Taking into account the finite coherence length in the sample and the focusing length of laser beams we estimate that the signals without NPs are generated from $\sim 300 \mu \mathrm{m}$ layer of bulk pyridine. Normalizing the ratios of the signals in Fig. 3e yields the enhancement factor of $\sim 10^{7}$. It is six orders of magnitude more than previously reported using femtosecond SECARS from a silver colloid ${ }^{10}$, and two orders of magnitude higher than from a structured surface ${ }^{12}$. Also, compared to femtosecond SECARS in ref. [12], it has a higher spectral resolution and lower nonresonant background. This value is closer to the theoretical estimate of $\sim 10^{10}$ for isolated $\mathrm{NPs}^{23}$.

Upon a further increase of the slit size the probe pulse duration approaches that of the original unshaped femtosecond pulse and all the spectra become congested (Fig. $3 \mathrm{~h}$ ). It is not possible to assign modes from any spectrum at one time delay. This drawback can be overcome by performing a time-resolved spectral measurement and analysis of the temporal traces. Fig. 3i shows a periodic quantum beating pattern for bulk pyridine without NPs (blue) and a more complicated beating pattern for pyridine with NPs (red). We fit these curves by a sum of three exponential functions and obtain vibrational dephasing rates which correspond to the previously resolved modes (the fitting procedure and the results (Table S1) are summarized in the Supporting Material). Decreasing the slit size yields better spectral resolution but larger nonresonant background due to the longer temporal duration of the probe pulse. The optimum is found for the $0.33 \mathrm{~mm}$ slit which shows narrow spectral lines and small background in Fig. 3e.

\section{Discussion}

Using tr-SECARS we obtained both spectral and temporal information from nanoscale volumes of pyridine in the vicinity of gold NPs. The spectra enable the characterization of the nature of pyridine complexes with other molecular species on the surface and the dynamics reveal their function. Using density functional theory (DFT) we calculated the equilibrium structures and Raman frequency shifts of various complexes of pyridine, water, and gold clusters. The simulation details are given in the Supporting Material. The calculated vibrational wave numbers of pyridine ring breathing (B) and triangle $(\mathrm{T})$ modes are listed in Tables S2-S4. Our simulation results show that in different complexes the triangle mode is within $\sim 3 \mathrm{~cm}^{-1}$ of the bulk pyridine (Fig. 1c). In contrast, the ring breathing mode shows a significant variation of shifts towards higher values. The shift due to pyridine-water hydrogen bond formation is smaller than the shift caused by the pyridine-gold interaction and matches well with the experimentally determined value of $\sim 7 \mathrm{~cm}^{-1}$. (The overall blue shift of the simulated signals with respect of the experimental value is due to quantum chemical approximations.) Therefore we conclude that the pyridine-water complex provides the most significant contribution to the observed tr-SECARS spectra (number 1 in Figs. 1a and 1c). Thus, tr-SECARS allows the detection of a small amount of water on the surface of gold nanoparticles. Previous studies reported similar line shifts observed from bulk mixtures of pyridine and water at silver surfaces ${ }^{1}$. In contrast, here we considered pure pyridine. Water is found as a small impurity on the surface of gold, which may have been adsorbed from the ambient air.

Here we report a first observation of nanoscale pyridine-water complexes using time-resolved surface-enhanced coherent Raman (tr-SECARS) spectroscopy. These complexes were not observed in the SERS spectra. This result highlights the differences between SERS and tr-SECARS. Different laser frequencies, powers, and acquisition times do not allow a direct comparison of the enhancement factors. We used a high power cw Nd:YAG laser to obtain the SERS signals in a reasonable time of $\sim 80 \mathrm{~s}$. This continuous perturbation might have caused degradation of the hydrogen-bonded complexes. On the other hand, tr-SECARS uses ultrashort laser pulses at $1 \mathrm{kHz}$ repetition rate with the total sub-nanosecond perturbation time of the system. This may have particular advantages for the investigation of fragile molecular complexes in biological systems which cannot sustain high-power SERS laser sources. Investigation of such biocomplexes at nanoscale requires depositing even more laser power to obtain good signals. Tr-SECARS provides a promising alternative route towards this goal. This method presents new promising opportunities for high resolution and high sensitivity characterization of nanoparticle surfaces and nanoscale biomolecular complexes. 
Tr-SECARS spectroscopy could be used to detect small amounts of toxic substances such as anthrax due to higher sensitivity compared with conventional coherent Raman techniques. Optimal pulse shapes could be found using open- ${ }^{24}$ and closed-loop ${ }^{25}$ coherent control methods applied on the nanoscale. Improvements of sample preparation could also increase enhancement factors. Resonant optical nanoantennas ${ }^{26}$ could be designed for optimal tr-SECARS signals controlled by pulse shaping ${ }^{27}$. Time-resolved tip-enhanced CARS spectroscopy ${ }^{7}$ and subwavelength microscopy could be realized by extension of the present approach. Spectral interpretation could be facilitated by using higher levels of theory and by direct modeling of the surface-enhanced CARS signals ${ }^{28}$. With further developments tr-SECARS could achieve ultrashort sub-picosecond signal acquisition and single shot real-time detection capabilities. Other related surface-enhanced nonlinear optical spectroscopic techniques could be developed based on the present approach ${ }^{29}$. Various coherent multidimensional optical spectroscopies could be implemented on the nanoscale that would benefit from the improved spectral and spatial resolution and higher sensitivity ${ }^{30,31}$. It would be possible to directly probe subwavelength vibrational and electronic response functions of complex artificial and biological systems in real time. Finally, tr-SECARS could be used as a new method for studying coherent dynamics of nanowater on the surface of biomolecules or in confined nanowires $^{32}$ for biomedical and nanofluidic applications.

1. Fleischmann, M., Hendra, P. J. \& McQuillan, A. J. Raman spectra of pyridine adsorbed at a silver electrode. Chem. Phys. Lett. 26, 163-166 (1974).

2. Kneipp, K., Moskovits, M. \& Kneipp, H. Surface-enhanced Raman Scattering: Physics And Applications (Springer, 2006).

3. Ru, E. C. L. \& Etchegoin, P. G. Principles of Surface-Enhanced Raman Spectroscopy: And Related Plasmonic Effects (Elsevier, 2008).

4. Schluecker, S. \& Kiefer, W. Surface Enhanced Raman Spectroscopy (John Wiley \& Sons, 2011).

5. Minck, R. W., Terhune, R. W. \& Rado, W. G. Laser-stimulated Raman effect and resonant four-photon interactions in gases $\mathrm{H}_{2}, \mathrm{D}_{2}$, and $\mathrm{CH}_{4}$. Appl. Phys. Lett. 3, 181-184 (1963).

6. Liang, E. J., Weippert, A., Funk, J. M., Materny, A. \& Kiefer, W. Experimental observation of surface-enhanced coherent anti-Stokes Raman scattering. Chem. Phys. Lett. 227, 115-120 (1994).

7. Ichimura, T., Hayazawa, N., Hashimoto, M., Inouye, Y. \& Kawata, S. TipEnhanced Coherent Anti-Stokes Raman Scattering for Vibrational Nanoimaging. Phys. Rev. Lett. 92, 220801 (2004).

8. Koo, T. W., Chan, S. \& Berlin, A. A. Single-molecule detection of biomolecules by surface-enhanced coherent anti-Stokes Raman scattering. Opt. Lett. 30, 1024-1026 (2005).

9. Addison, C. J., Konorov, S. O., Brolo, A. G., Blades, M. W. \& Turner, R. F. B. Tuning Gold Nanoparticle Self-Assembly for Optimum Coherent Anti-Stokes Raman Scattering and Second Harmonic Generation Response. J. Phys. Chem. C 113, 3586-3592 (2009)

10. Namboodiri, V. et al. Surface-enhanced femtosecond CARS spectroscopy (SECARS) on pyridine. Vib. Spectrosc. 56, 9-12 (2011).

11. Wang, Y., Lin, C. Y., Nikolaenko, A., Raghunathan, V. \& Potma, E. O. Four-wave mixing microscopy of nanostructures. Adv. Opt. Photon. 3, 1-52 (2011).

12. Steuwe, C., Kaminski, C. F., Baumberg, J. J. \& Mahajan, S. Surface Enhanced Coherent Anti-Stokes Raman Scattering on Nanostructured Gold Surfaces. Nano Lett. 11, 5339-5343 (2011).

13. Hayazawa, N., Ichimura, T., Hashimoto, M., Inouye, Y. \& Kawata, S. Amplification of coherent anti-Stokes Raman scattering by a metallic nanostructure for a high resolution vibration microscopy. J. Appl. Phys. 95, 2676-2681 (2004).

14. Schluecker, S., Singh, R. K., Asthana, B. P., Popp, J. \& Kiefer, W. HydrogenBonded Pyridine-Water Complexes Studied by Density Functional Theory and Raman Spectroscopy. J. Phys. Chem. A 105, 9983-9989 (2001).

15. Schluecker, S. et al. Vibrational Dynamics in Hydrogen-Bonded (Pyridine + Water) Complexes Studied by Spectrally Resolved Femtosecond CARS. Z. Phys. Chem. 216, 267 (2002).
16. Berg, E. R., Freeman, S. A., Green, D. D. \& Ulness, D. J. Effects of Hydrogen Bonding on the Ring Stretching Modes of Pyridine. J. Phys. Chem. A 110, 1343413446 (2006).

17. Daniel, M. C. \& Astruc, D. Gold Nanoparticles: Assembly, Supramolecular Chemistry, Quantum-Size-Related Properties, and Applications toward Biology, Catalysis, and Nanotechnology. Chem. Rev. 104, 293-346 (2004).

18. Elghanian, R., Storhoff, J. J., Mucic, R. C., Letsinger, R. L. \& Mirkin, C. A. Selective Colorimetric Detection of Polynucleotides Based on the Distance-Dependent Optical Properties of Gold Nanoparticles. Science 277, 1078-1081 (1997).

19. Le Ru, E. C. \& Etchegoin, P. Single-Molecule Surface-Enhanced Raman Spectroscopy. Annu. Rev. Phys. Chem. 63 (2012).

20. Scully, M. O. et al. FAST CARS: Engineering a laser spectroscopic technique for rapid identification of bacterial spores. Proc. Natl. Acad. Sci. USA 99, 10994-11001 (2002).

21. Pestov, D. et al. Optimizing the Laser-Pulse Configuration for Coherent Raman Spectroscopy. Science 316, 265-268 (2007).

22. Pestov, D. et al. Single-shot detection of bacterial endospores via coherent Raman spectroscopy. Proc. Natl. Acad. Sci. USA 105, 422-427 (2008).

23. Chew, H., Wang, D. S. \& Kerker, M. Surface enhancement of coherent anti-Stokes Raman scattering by colloidal spheres. J. Opt. Soc. Am. B 1, 56-66 (1984).

24. Aeschlimann, M. et al. Optimal open-loop near-field control of plasmonic nanostructures. New. J. Phys. 14, 033030 (2012).

25. Aeschlimann, M. et al. Spatiotemporal control of nano-optical excitations. Proc. Natl. Acad. Sci. USA 107, 5329-5333 (2010).

26. Mühlschlegel, P., Eisler, H.-J., Martin, O. J. F., Hecht, B. \& Pohl, D. W. Resonant Optical Antennas. Science 308, 1607-1609 (2005).

27. Huang, J. S., Voronine, D. V., Tuchscherer, P., Brixner, T. \& Hecht, B. Deterministic spatiotemporal control of optical fields in nanoantennas and plasmonic circuits. Phys. Rev. B 79, 195441 (2009).

28. Parkhill, J. A., Rappoport, D. \& Aspuru-Guzik, A. Modeling Coherent Anti-Stokes Raman Scattering with Time-Dependent Density Functional Theory: Vacuum and Surface Enhancement. J. Phys. Chem. Lett. 2, 1849-1854 (2011).

29. Genevet, P. et al. Large Enhancement of Nonlinear Optical Phenomena by Plasmonic Nanocavity Gratings. Nano Lett. 10, 4880-4883 (2010).

30. Mukamel, S. Principles of nonlinear optical spectroscopy (Oxford, New York, 1995).

31. Aeschlimann, M. et al. Coherent Two-Dimensional Nanoscopy. Science 333, 1723-1726 (2011).

32. Lee, Y., Martin, C. D., Parise, J. B., Hriljac, J. A. \& Vogt, T. Formation and Manipulation of Confined Water Wires. Nano Lett. 4, 619-621 (2004).

\section{Acknowledgements:}

We gratefully acknowledge the support of the National Science Foundation Grant EEC-0540832 (MIRTHE ERC), the Office of Naval Research, the Army Research Office (grant No. W911NF-07-1-0475), the American Chemical Society Petroleum Research Fund (ACS PRF 50645-DNI6), and the Robert A. Welch Foundation (A-1261 and A-1547). We thank the Herman F. Heep and Minnie Belle Heep Texas A\&M University Endowed Fund held/administered by the Texas A\&M Foundation for financial support. We thank Charles Ballmann, Guowan Zhang, Wenlong Yang and Ziyun Di for help with experiments and Hui Xia, Xi Wang, Patrice Genevet, Raymong Ooi and Phil Hemmer for helpful discussions.

\section{Author contributions:}

D.V.V., A.M.S., X.H. and K.W. performed the experiments; E.M. and S.E.W. performed the simulations; D.V.V., A.M.S., X.H., K.W., P.K.J., G.W., A.V.S. and M.O.S. analyzed the data and wrote the paper; A.V.S. and M.O.S. supervised the project.

\section{Additional information}

Supplementary information accompanies this paper at http://www.nature.com/ scientificreports

Competing financial interests: The authors declare no competing financial interests.

License: This work is licensed under a Creative Commons Attribution-NonCommercial-NoDerivs 3.0 Unported License. To view a copy of this license, visit http://creativecommons.org/licenses/by-nc-nd/3.0/

How to cite this article: Voronine, D.V. et al. Time-Resolved Surface-Enhanced Coherent Sensing of Nanoscale Molecular Complexes. Sci. Rep. 2, 891; DOI:10.1038/srep00891 (2012). 